\title{
Gate Recognition and Reconstruction for DARPA Robotics Challenge using Bayesian Classifier Optimized by Mahalanobis Distance
}

\author{
Andrés Cela \\ Department of Automation and \\ Industrial Control \\ National Polytechnic School \\ Quito - Ecuador \\ andres.cela@epn.edu.ec
}

\author{
Luis M. Bergasa \\ Department of Electronics \\ University of Alcalá \\ Alcalá de Henares \\ España \\ bergasa@depeca.uah.es
}

\author{
Roberto Arroyo \\ Department of Electronics \\ University of Alcalá \\ Alcalá de Henares \\ España \\ rarroyo@depeca.uah.es
}

\begin{abstract}
Darpa Robotics Challenge contest has different goals that a humanoid robot must achieve in order to reach the next contest stages. The robot has different sensors, such as cameras, gps, etc. In this paper we do not deal with goals but just how to detect the gates based on the mono camera images. We describe how to recognize the posts of the goal based on a simple threshold algorithm. We have used these data for implementing a Bayesian classifier optimized by the Mahalanobis distance for classifying the pixels by color because each post has different color. Then, a Hough lines detector has been used for detecting the lines in the post. Thus, posts are represented by lines. Once detected the posts, the Euclidean distance is used for matching the corner posts with their corresponding pair. Thereby, gate is represented by three interconnected lines. Finally, we have calculated a line from the robot to the center of the gate in order to give the robot a path for arriving to the goal. As an additional work we have detected the pixels of the gate number which is in the gates corner.
\end{abstract}

Keywords-Darpa Robotic Challenge, color detection, Bayesian classifier.

\section{INTRODUCTION}

Darpa Robotics Challenge (DRC) is an open contest which presents three different simulated scenarios and each one has different goals like get into a vehicle and drive it, walk trough unknown environments and manipulate some objects. In this paper we describe how to detect the gates which are in these scenarios. Firstly, the robot must recognize a gate made of three posts: the upper post, the left post and the right post, each one has different color, green, blue and red respectively. Once the robot has recognized the goal, it must know its position in order to go to the gate and pass through it to the next gate. The gates have a number in the upper corner which indicates the order to pass the gates. Although, the robot needs to recognize the gate number, this paper is however will just explain the gate recognition, reconstruction and number color detection.

Different research centers of the world participate in this contest whose final goal is to generate groundbreaking research. Thus, in the future a robot will be able to perform tasks that are dangerous for people. [1]
The color of the gates have a simple texture and it does not allow to use a classifier which uses this variable [2], instead it uses the color information. In this paper we propose to use a Bayesian Classifier (BC) in order to indentify the three posts by color. $\mathrm{BC}$ is an optimal classification and it is fast to work with few variables. Sometimes it is combined with other kind of classifiers in order to get a stronger algorithm like the presented in [3] and [4], these pretend to improve the $\mathrm{BC}$ when the model does not have a normal distribution [4].

Color recognition has been studied in many ways but it depends on the quality of color. The authors in [5] use a kmeans method for recognizing the color of the clothes. It achieves an $80 \%$ of success. The color recognition in [6] is done by a comparison between primaries color and the color object, this uses a simple classifier to identify the color. The color model can do easier the color recognition, but with a combination of the models color the results can be better, as is described in [7].

The color classification can be done using different variables like the RGB channels or the HSV channels. HSV channels are used for many applications to classify color because it is possible to obtain enough information about color in just two channels, while in RGB three channels are necessary. In this paper we propose to use HS variables.

In this paper, our proposal is to use a Bayesian Classifier (BC) in order to recognizing the post colors.

Besides, Mahalanobis Distance (MD) and BC with normal distribution use a common covariance matrix to calculate the distance from the element to the main of the set. $\mathrm{BC}$ uses this matrix in a normal function and its implementation needs more resources to calculate it. MD uses this matrix directly for calculating the distance and its implementation is easier and faster because in the algorithm does not appear exponential functions like in $\mathrm{BC}$.

On the other hand, color detection is the first step in this work. A simple classifier based on threshold in the RGB channels is used for detecting the posts by color. Then this information is used for designing and training a $\mathrm{BC}$ optimized by MD based on HSV channels. The lines, which represent the posts, are obtained by Hough lines detector [8]. Once detected the posts it is necessary to identify what is the left one and what the right one. Next step is to built the gate 
and calculate the angle that robot needs to turn to go to the gate. The nearest neighbor method based on a Euclidean Distance (ED) is used for matching the corner posts with their corresponding pair.

Finally, a reconstructed gate and a line with the orientation angle of the robot are shown on the screen. This information will be used by another group who directs the robot to the gate.

Section 2 describes the proposed algorithm which is done in 4 phases. Section 3 shows the results. The conclusions of this work are in section 4.

\section{PROPOSED ALGORITHM}

The proposed algorithm has four phases. The first one is the Threshold detection, then a BC is training based on data of the first phase, the third phase is the post lines detection based on Hugh detector and gate reconstruction and last phase is the robot orientation estimation.

\section{A. Post detection based on Threshold}

The robot has a stereo camera with resolution of $800 \times 800$ for each image. We have used just the left image for detecting gates. The images are in RGB model.

The gate has three posts and a specific orientation. In the front view the upper post is green, the right one is red and the left one is blue.

In this phase we have defined a threshold for each color post. The threshold detection is based on heuristic information of the posts color values in RGB channels. The threshold values for each color have been obtained by inspecting the RGB values of some pixels of each post. Thus, we have noticed that color posts have high values in its respective RGB color channel. Thereby, red color is approximately three times higher in red channel than in other channels. Thresholds for red color are: higher than 96 in red channel and fewer than 30 in green and blue channels. In this manner we have defined the ranges of color for each post. Thresholds are described in Table 1 .

TABLE I. RANGES OF POSTS COLOR THRESHOLD IN RGB CHANNELS

\begin{tabular}{|c|c|c|c|}
\hline Post & R - CH & G - CH & B - CH \\
\hline Red & $>96$ & $<30$ & $<30$ \\
\hline Green & $<30$ & $>96$ & $<30$ \\
\hline Blue & $<30$ & $<30$ & $>96$ \\
\hline
\end{tabular}

We have obtained a first color detection with a simple classifier based on thresholds. The true positives are the pixels that satisfy the ranges on Table 1 . The true negatives are set in white. Fig. 1 shows the posts detection based on thresholds. The red pixels of the two gates have been detected, in (b) the green pixels have been detected and in (c) the blue pixels. All these pixels have been used for training the Bayesian classifier.

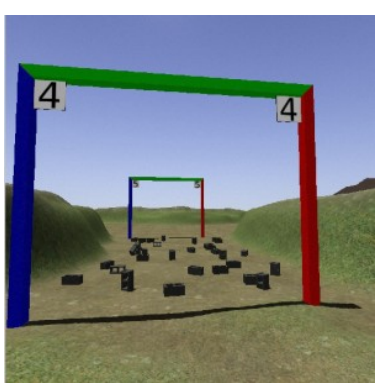

(a)

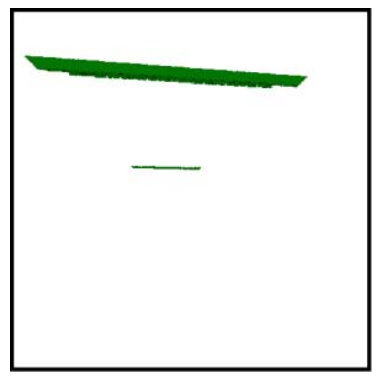

(c)

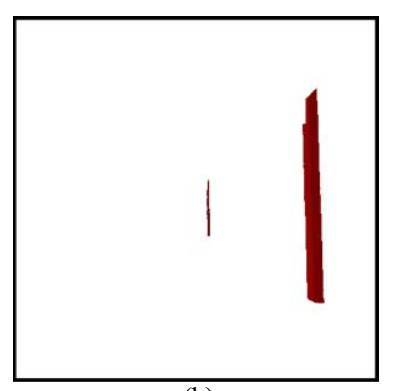

(b)

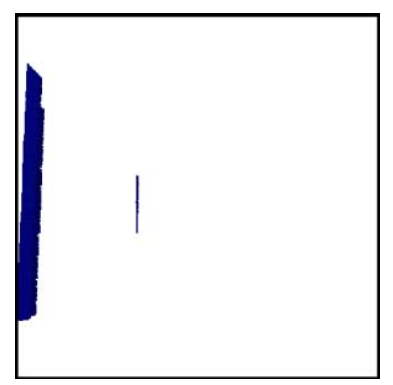

(d)
Figure 1. Color detection based on threshold values. (a) Original image. (b) Red detection. (c) Green detection (d) Blue detection.

\section{B. Training and testing the Bayesian Classier}

Three images have been considered for designing the Bayes classifier. The first one corresponds to the first image that the robot sees when it is in the start room. Here is the first gate which indicates the start place. The second image shows a gate in a special view where the red post has shadow. In the third image there is a complete gate and it has shadows in all three posts, this is shown in Fig. 1 (a). These images have been used for designing the BC because they have special characteristics of color, shadows and brightness.

Once detected the posts colors, the image of each one is turned to HSV model. The $\mathrm{H}$ and $\mathrm{S}$ channels have been used because these channels give information about color, while the $\mathrm{V}$ channel gives information about brightness which is not important in this research.

Bayes' formula is the general way to understand BC. It converts the prior $P\left(w_{i}\right)$ probability to the posterior probability $P\left(w_{i} \mid x\right)$. The $p\left(x \mid w_{i}\right)$ function is the likelihood of $w_{i}$ respect to $x$ and $i$ is the number of categories [9], as it is described in (1).

$$
P\left(w_{i} \mid x\right)=\frac{p\left(x \mid w_{i}\right) \cdot P\left(w_{i}\right)}{p(x)}
$$


The prior probability for each color is $1 / 3$ because the pixel could be whatever of three colors. The evidence factor $p(x)$ is the same for all categories and it can be viewed as a merely scale factor to normalize to 1 the posterior probability. A normal distribution is used for representing each $\boldsymbol{w}$ set. Finally, the prior probability function is shown in (2) which has a normal shape.

$$
P(w \mid u, \xi)=1 /\left(\xi .(2 \pi)^{1 / 2}\right) \cdot \exp \left(-(x-u)^{2} /(2 . \xi)\right)
$$

The set pixels of each color in the three HSV images have been used for calculating the parameters of BC, these are, the covariance matrix $\boldsymbol{\xi}$ and the mean matrix $\boldsymbol{u}$ for each color. The $\boldsymbol{x}$ vector corresponds to the characteristics of each pixel in HSV model and it is a two dimensional vector.

The BC can be optimized by logarithmic expressions to MD classifier [10] as it is showed in (3).

$$
P(w \mid u, \xi)=-\ln \left(2 \pi^{1 / 2}|\xi|^{1 / 2}\right)-(x-u)^{T} \xi^{-1}(x-u) / 2
$$

Where the first component is a constant and the second one is known as MD. The $\boldsymbol{w}$ color with the highest probability will be assigned to the pixel.

The mean has been calculated as the mean of all pixels by color of the three images described above. The covariance matrices have been calculated as the matrix covariance of each color of all pixels of the three images.

Table 2 shows the normal distribution parameters for each color. The $\mathrm{BC}$ optimized by $\mathrm{MH}$ has been testing using these parameters.

The $\mathrm{BC}$ result is shown in Fig. 2, where there are two images classified. The first one corresponds to the start of the stage and the second image to the middle of the stage. The first image has red color in the wall. These pixels have also been classified like red pixels, as it can be seen in Fig. 2(a) and (b), they do not against the post but the wall instead. In (b) and (f), the red detection has also error because some green pixels have been detected like red ones. These errors will be reduced in the next phases.

TABLE II. NORMAL DISTRIBUTION PARAMETERS

\begin{tabular}{|c|c|c|}
\hline Parameter & Covariance & Mean \\
\hline \multirow{2yn}{*}{ Red } & {$\left[\begin{array}{ll}0.2164 & 0.0007 \\
0.0007 & 0.0012\end{array}\right]$} & {$\left[\begin{array}{l}0.3233 \\
0.9686\end{array}\right]$} \\
\hline Green & {$\left[\begin{array}{cc}0.0002 & -0.0002 \\
-0.0002 & 0.0015\end{array}\right]$} & {$\left[\begin{array}{l}0.3360 \\
0.8893\end{array}\right]$} \\
\hline Blue & {$\left[\begin{array}{cc}3.72 \mathrm{e}-5 & 2.51 \mathrm{e}-5 \\
2.51 \mathrm{e}-5 & 0.0036\end{array}\right]$} & {$\left[\begin{array}{l}0.6649 \\
0.8855\end{array}\right]$} \\
\hline
\end{tabular}

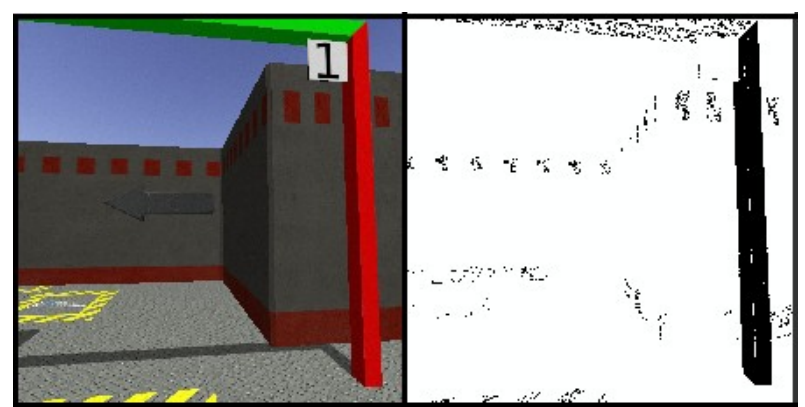

(a)

(b)

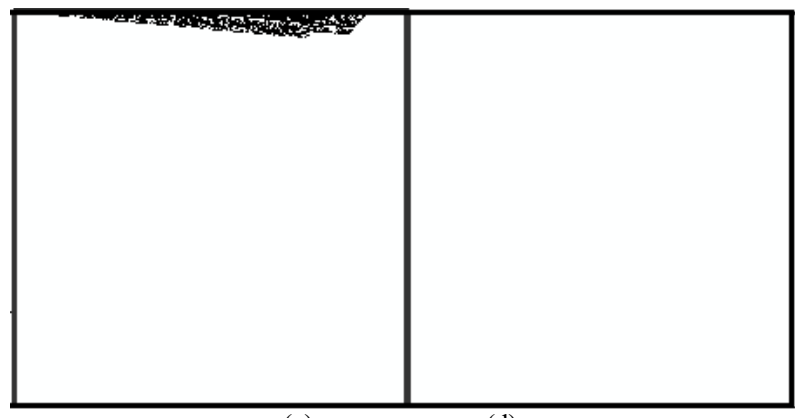

(c)

(d)

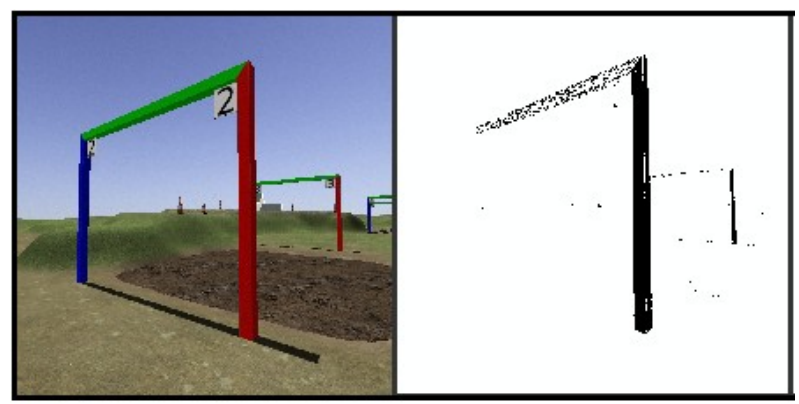

(e)

(f)

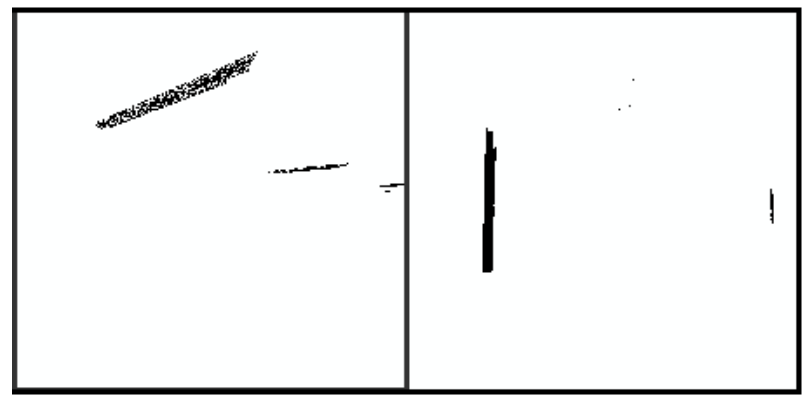

(g)

(h)

Figure 2. Color detection based on Bayesian Classifier (a), (e) Original images in the start and in the middle of the stage respectively. (b), (f) Red classification. (c), (g) Green classification. (d), (h) Blue classification. 


\section{Gate Reconstruction}

The Hough Lines Detector (HLD) allows recognizing the lines in an image. In OpenCv, HLD generates a set of possible lines [11]. We have discriminated the false lines based in characteristics like dimensions and slope. Due to the standing position of the robot, images are parallels to the vertical posts and, if the slope of red and blue lines is near to the horizontal, "2", then the line is discarded. On the other hand, if a line is shorter than $10 \%$ of the Image Size in "Y" Axes (ISYA) and there is other line higher, then the shorter one is discarded.

The higher lines are taken to form the gate. The gate reconstruction is based on ED. The first point and the last point have been called Point 1 (P1) and Point 2 (P2) respectively taken from top down for red and blue lines and from left to right for the green line. If the distance between $\mathrm{P} 1$ in blue line and P1 in green line is shorter than $10 \%$ ISYA then these two lines are pairs and correspond to left gate corner, but if the nearer pixel is $\mathrm{P} 2$ green then it is the right gate corner, this means that the robot is on the other side of the gate.

On the other hand, If the distance between P1 in red line and P2 in green line is shorter than 10\% ISYA, then these pixels are pairs and correspond to right gate corner, but if $\mathrm{P} 1$ green is nearer it is the left gate corner instead. With this algorithm the robot recognizes the side of the gate and also reconstructs the gate.

If the distance between the points described above is higher than $10 \%$ ISYA, the second highest line is evaluated.

In some cases can appear an incomplete gate but the reconstruction is complete.

Fig. 3 shows in (a) the lines detection in yellow and in (b) the gate reconstruction in black. The detected lines in yellow are generally not together. A media point is calculated between the points that form that right corner. This point became the P2 in green line and P1 in red line as it is shown in Fig. 3 (b). Although the blue post is not in the original image, the gate reconstruction has a black line on the left which corresponds to the blue post. This line has been predicted using the positions of red and green lines.

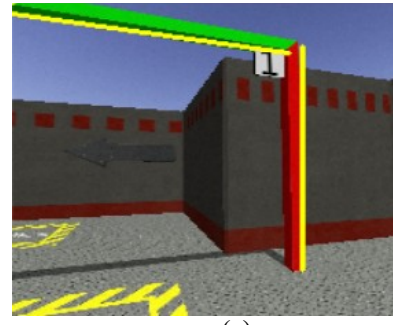

(a)

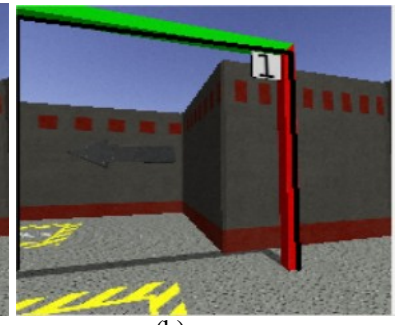

(b)
Figure 3. (a) Lines detection. (b) Gate reconstruction.

\section{Orientation Estimation}

The robot has a camera on its head and it senses the robot frontal plane, like its eyes. With this consideration, the middle pixel in the last row in the image can be considered as the initial point for measuring the angle between the robot frontal plane and the gate plane formed by the three posts.

The line between pixels $\mathrm{P} 2$ in red line and $\mathrm{P} 2$ in blue line is the projection on the floor of the gate plane, the line L1 in Fig. 4. The last row, line L2, in the image is the projection of the robot front plane. The complementary angle $\alpha$ to the angle formed by these two lines is the deviation of the robot. A line that connects the middle points of these lines is considered as a shortest path to go to the gate. In Fig. 4 the line between the $\mathrm{Pa}$ and $\mathrm{Pb}$ points is the shortest path. But in some stages this path is not used because there are areas with obstacles.

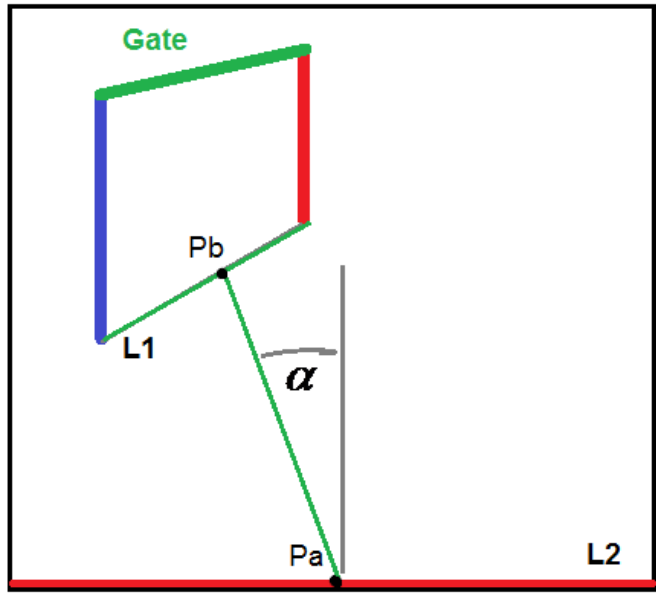

Figure 4. Path estimation.

The $\alpha$ angle can be calculated using (4).

$$
\alpha=90-\operatorname{tg}^{-1}((I S Y A-P b) /(P a-P b))
$$

The robot also needs to recognize the gate number, but in this work we have not recognized the number but color number instead. The number recognition is being done by other research group. In all images that have a gate, the number is in the middle upper section, because it is in the gate corners, as shown in the images.

Fig. 5 shows a sequence of images where a completed algorithm has been applied. The green lines represent the orientation and the path to the gate.

These sequences have been taken in the exit of the initial room in the stage 2 . In the sequence the robot is crossing the gate 1. The gate is detected while it appears on the image, but if the gate disappears, a message will indicate this event, like in fifth image. The last part of the sequence is in the exit of initial room and gate 2 has been recognized in the front of robot. 

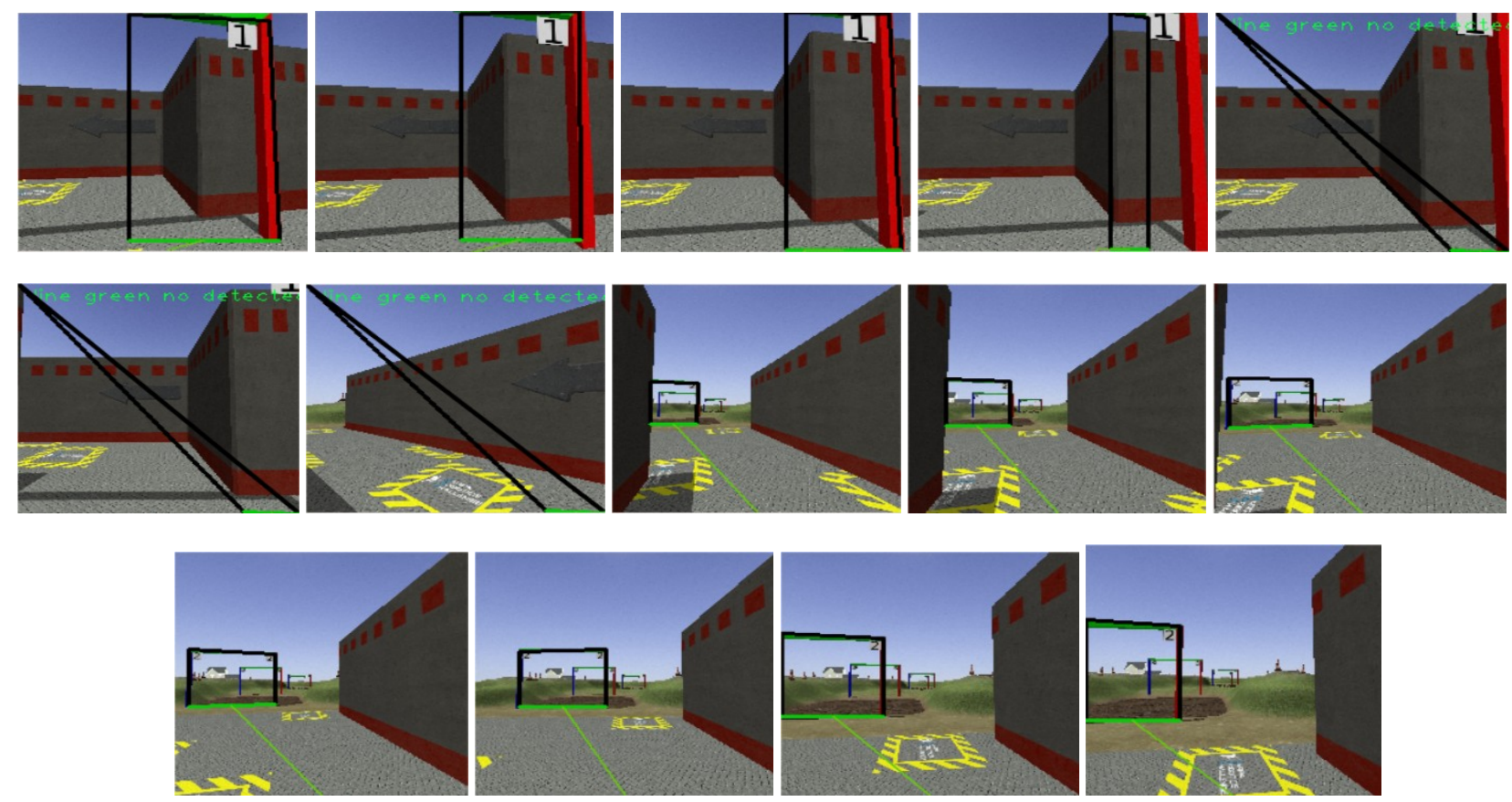

Sequence

Figure 5. Gate detection.

The method for recognizing the color of the number also is based on threshold. The color number is in black over a white background. The threshold for black color is RGB $(5,5,5)$, this means that pixels with a RGB color values higher than the thresholds values are not considered like number pixels.

Fig. 6 shows the color number detection in Fig. 3. All pixels that do not correspond to the number are in white.

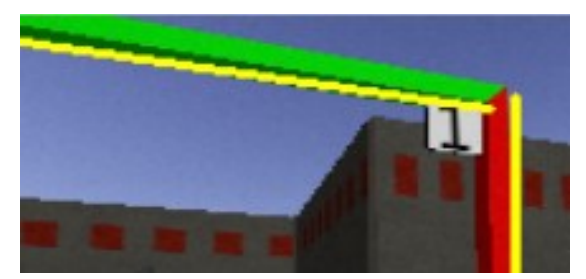

(a) Image

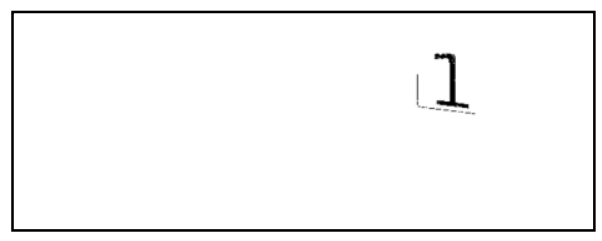

(b) Pixels of the number.

Figure 6. Color number detection.

\section{EXPERIMENTAL RESULTS}

The test has been done in a stage where the robot made a journey in order to get a long sequence of images with different views of gates. This sequence has 864 images and detection results are shown in Table 3 where the column "Gate" indicates when in the image there is a gate, "Without" indicates that in the image there is not a gate and the last row indicates that the gate is smaller than 10\% ISYA. The column "Images" indicates how many images there are for each type of gate. The column "Gate Det" indicates the results of gate detection. The next column indicates the orientation detection and last column indicates the color number detection.

TABLE III. RESUlts of Detection IN A Long SEQuence

\begin{tabular}{|c|c|c|c|c|}
\hline Gate & Images & Gate Det. & Orien. Det. & Number Det. \\
\hline Normal & 808 & $81.7 \%$ & $90.85 \%$ & $100 \%$ \\
\hline Without & 49 & $0.8 \%$ & $0 \%$ & $0 \%$ \\
\hline Small & 7 & $0 \%$ & $0 \%$ & $0 \%$ \\
\hline
\end{tabular}

Results show that this algorithm detects and recognizes $81.7 \%$ of images that have gates. When image does not have gates the error of recognition is $0.8 \%$. When gate is smaller than $10 \%$ ISYA gate is not recognized. The orientation is well estimated in $90.85 \%$ and the pixels number detection is recognized in all images of the sequence. 


\section{CONCLUSIONS}

We have proposed an algorithm for recognizing gates in the stages of DARPA contest. The first phase classifies the colors of the gate which has three stakes with different colors. First of all, a classifier based on threshold was designed using a basic color filter. Although this one yields good results, it is not robust to color changes or shadows.

A Bayesian classifier was designed based on the pixels of the previous phase. The classifier was designed for two variables, the $\mathrm{H}$ and $\mathrm{S}$ channels, instead RGB channels. The parameters for the normal function which is used in the classifier have been calculated using three images that contain shadows, brightness and gates in different views.

The Bayesian classifier with normal distribution can be optimized using logarithmic functions. It allows obtaining a shorter expression, as shown in (3), which is faster to compute.

Although the robot has a stereo camera, we have demonstrated in this work that a mono camera is enough for recognizing gates and orienting robot.

The Hough transform is a good solution for detecting the posts in the gate. This filter is already implanted in OpenCv software. Whereby, our work consists in selecting the best lines of each post which form the reconstructed gate.

Finally, the path between robot and gate allows optimizing time for reaching the gate, although this is not necessary the final path since other research group will use this one for constructing the real path. The detection of color number is an important advance for other research groups that will recognize the number in order to know what gate is and how long is the end of the stage.

As it is shown in Table 3, the detection error in normal images which contains gates is lower than $10 \%$ and error in orientation is similar. The false detection in images that do not contain gates is $1 \%$.

Thus, we conclude that this algorithm can be used for detecting and reconstructing gates in DRC. This work has been used for DARPA contest in simulated environments.

\section{REFERENCES}

[1] «DARPA DRC | DARPA Robotics Challenge Home». [En línea]. Disponible en: http://www.theroboticschallenge.org/. [Accedido: 28ago-2013].

[2] M. S. Neofytou, V. Tanos, M. S. Pattichis, C. S. Pattichis, E. C. Kyriacou, y S. Pavlopoulos, «Color Based Texture - Classification of Hysteroscopy Images of the Endometrium», en 29th Annual International Conference of the IEEE Engineering in Medicine and Biology Society, 2007. EMBS 2007, 2007, pp. 864-867.

[3] Z. Wu, B. Zhang, Y. Zhu, W. Zhao, y Y. Zhou, «Transformer Fault Portfolio Diagnosis Based on the Combination of the Multiple Bayesian Classifier and SVM», en 2009 International Conference on Electronic Computer Technology, 2009, pp. 379-382.

[4] Q. Liang, «MPEG VBR video traffic classification using Bayesian and nearest neighbor classifiers», en IEEE International Symposium on Circuits and Systems, 2002. ISCAS 2002, 2002, vol. 2, pp. II-77II- 80 vol.2.

[5] X. Zheng y N. Liu, «Color recognition of clothes based on k-means and mean shift», en 2012 IEEE International Conference on Intelligent Control, Automatic Detection and High-End Equipment (ICADE), 2012, pp. 49-53.

[6] M. Yang, G. Han, X. Li, X. Zhu, y L. Li, «Vehicle color recognition using monocular camera», en 2011 International Conference on Wireless Communications and Signal Processing (WCSP), 2011, pp. $1-5$.

[7] T. Liu, H. Guo, y Y. Wang, «A New Approach for Color-Based Object Recognition with Fusion of Color Models», en Congress on Image and Signal Processing, 2008. CISP '08, 2008, vol. 3, pp. 456460.

[8] R. O. Duda, P. E. Hart, y D. G. Stork, Pattern Classification. John Wiley \& Sons, 2012.

[9] Y.-H. Cai, «The comparative study of different Bayesian classifier models», en 2010 International Conference on Machine Learning and Cybernetics (ICMLC), 2010, vol. 1, pp. 309-313.

[10] A. Cela, F. Sánchez, «Unsupervised Classifier for Dynamic Systems Based on ISODATA», Acepted Publ.

[11] G. Bradski y A. Kaehler, Learning OpenCV: Computer Vision with the OpenCV Library. O'Reilly, 2008. 